DIE WIRTSCHAFTLICHEN UNTERNEHMUNGEN DER SS 
SCHRIFTENREIHE

DER VIERTELJAHRSHEFTE FÜR ZEITGESCHICHTE NUMMER 7

Im Auftrag des Instituts für Zeitgeschichte

herausgegeben von Hans Rothfels und Theodor Eschenburg Redaktion: Martin Broszat

DEUTSCHE VERLAGS-ANSTALT STUTTGART 
ENNO GEORG

\section{DIE WIRTSCHAFTLICHEN UNTERNEHMUNGEN DER SS}

DEUTSCHE VERLAGS-ANSTALT

STUTTGART 
(c) 1963 Deutsche Verlags-Anstalt GmbH., Stuttgart. Gesetzt aus der Monotype Walbaum-Antiqua. Gesamtherstellung: Deutsche Verlags-Anstalt GmbH., Stuttgart.

Printed in Germany 


\section{INHALT}

VERZEICHNIS DER ABKÜRZUNGEN UND DIENSTGRADE . . . . . . . . 7

VORWORT . . . . . . . . . . . . . . . . . . . . 9

I. DIE ERSTEN WIRTSCHAFTLICHEN UNTERNEHMUNGEN DER SS . . . 12

1. Die Werkstätten-Betriebe in den frühen Konzentrationslagern . . . . 12

2. Die Unternehmungen des Persönlichen Stabes des Reichsführers-SS . $\quad 14$

A. Nordland-Verlag $\mathrm{GmbH}$. . . . . . . . . . . . . . . . . . 15

B. Porzellan-Manufaktur Allach-München $\mathrm{GmbH}$. . . . . . . . . 16

C. F. F. Bauer $\mathrm{GmbH}$. . . . . . . . . . . . . . . . . . . . 18

D. Anton Loibl $\mathrm{GmbH}$. . . . . . . . . . . . . . . . . . . . 19

E. Gemeinnützige Wohnungs- und Heimstätten GmbH, Dachau . . . 19

F. Spargemeinschaft-SS . . . . . . . . . . . . . . . . . . . . 20

G. Gesellschaft zur Förderung und Pflege Deutscher Kulturdenkmäler e. V. . . . . . . . . . . . . . . . . . . . . . . . . 21

H. Externsteine-Stiftung . . . . . . . . . . . . . . . . . . . 23

I. König Heinrich I.-Gedächtnis-Stiftung . . . . . . . . . . . . 23

II. DAS WIRTSCHAFTS-VERWALTUNGSHAUPTAMT DER SS UND SEINE VORLÄUFER . . . . . . . . . . . . . . . . . . . . . . . . . 25

1. Die organisatorische Entwicklung . . . . . . . . . . . . . . . . 25

2. Die einzelnen Amtsgruppen des WVHA . . . . . . . . . . . . . 33

III. DIE GROSSEN HÄFTLINGSUNTERNEHMUNGEN . . . . . . . . . . . 42

1. Deutsche Erd- und Steinwerke GmbH (DEST) . . . . . . . . . . 42

A. Die Steinbrüche und Granitwerke der DEST . . . . . . . . . . 44

B. Die Ziegel-, Kies- und Baustoffwerke der DEST . . . . . . . . . . 47

C. Organisatorische Leitung und geschäftliche Entwicklung . . . . . 54

D. Umstellung auf Rüstungsproduktion . . . . . . . . . . . . . 56

2. Deutsche Ausrüstungswerke $\mathrm{GmbH}(\mathrm{DAW})$. . . . . . . . . . . 58

3. Deutsche Versuchsanstalt für Ernährung und Verpflegung GmbH . . 62

4. Gesellschaft für Textil- und Lederverwertung $\mathrm{GmbH}$. . . . . . . 66 
IV. DIE AUSBREITUNG DER SS-WIRTSCHAFT WÄHREND DES KRIEGES

1. Gründung der Dachgesellschaft „Deutsche Wirtschaftsbetriebe“ DWB

2. Der Erwerb von Mineralwasser-, Keramik- und Möbelfirmen im Sudetenland und „Protektorat". . . . . . . . . . . . . . . . . . . 72

3. Die Baustoffwerke in den Ostgebieten . . . . . . . . . . . . . . 83

4. Die Wirtschaftsbetriebe der SS im Generalgouvernement (Ostindustrie $\mathrm{GmbH})$.

5. Die wirtschaftliche Betätigung der SS in den besetzten russischen Gebieten . . . . . . . . . . . . . . . . . . . . . . . . . . . 99

6. Neue Wirtschaftsunternehmungen im Altreichsgebiet . . . . . . . 101

V. DER ARBEITSEINSATZ DER HÄFTLINGE . . . . . . . . . . . . . 107

VI. ORGANISATION UND GESCHÄFTSFÜHRUNG . . . . . . . . . . . . . 119

1. Die Amtsgruppe W . . . . . . . . . . . . . . . . . . . . . . 119

2. Die leitenden Organe und Personen . . . . . . . . . . . . . . 124

VII. FINANZIERUNG UND VERMÖGENSRECHTLICHE STELLUNG DER SS-UNTERNEHMUNGEN . . . . . . . . . . . . . . . . . . . . . . . 133

1. Kapitalquellen und Gewinn . . . . . . . . . . . . . . . . . . 133

2. Die vermögensrechtlichen Verhältnisse . . . . . . . . . . . . . 138

SCHLUSSBETRACHTUNG . . . . . . . . . . . . . . . . . . . . . . 143

PERSONENVERZEICHNIS . . . . . . . . . . . . . . . . . . . . . . 147

VERZEICHNIS DER FIRMEN, ÄMTER UND INSTITUTIONEN . . . . . . 150 
Affid.

AG

DAW

DEST

DWB

eGmbH

e.V.

F. IV

GBI

Gestapo

GG

$\mathrm{GmbH}$

HTO

IMT

KI.

$\Lambda G$

$\mathrm{NI}$

NO

NSDAP

Osti

Prot. IY. IV

PS

RFSS

RKF

RMI

RSHA

RuSHA

SA

SD

Sipo

SS

Vert. Jok.

$V$ - und W-Hauptamt

WVHA
Affidavit (eidesstattliche Erklärung)

Aktiengesellschaft

Deutsche Ausrüstungswerke $\mathrm{GmbH}$

Deutsche Erd- und Steinwerke $\mathrm{GmbH}$

Deutsche Wirtschaftshetriebe $\mathrm{GmbH}$

eingetragene Genossenschaft mit beschränkter Haftung

eingetragener Verein

Fall IV (Prozeß gegen Pohl und andere)

Generalbauinspektor für die Reichshauptstadt

Geheime Staatspolizei

Generalgouvernement

Gesellschaft mit beschränkter Haftung

Haupttreuhandstelle Ost

International Military Tribunal (Der Prozeß gegen die Hauptkriegsverbrecher vor dem Internationalen Militärgerichtshof in Nürnberg)

Konzentrationslager (KL war die offirielle Abkürzung. Die im Volksmund entstandene Abkürzung KZ ist erst seit 1945 allgremein üblich geworden.)

NG-Serie der Nürnberger Akten (NG = Nazi Government)

NI-Serie der Nürnberger Akten (NI = Nazi Industry)

NO-Serie der Nürnberger Akten (NO = Nazi Organisation)

Nationalsozialistische Deutsche Arbeiterpartei

Ostindustrie $\mathrm{GmbH}$

Sitzungsprotokoll des Pohl-Prozesses (Fall IV)

PS-Serie der Nümberger Akten (PS = Paris Storey)

Reichsführer-SS

Reichskommissar für die Festigung deutschen Volkstums

Reichsmark

Reichssicherheitshauptamt

Rasse- und Siedlungshauptamt-SS

Sturmabteilung

Sicherheitsdienst des Reichsführers-SS

Sicherheitspolizei

Schutzstaffel

Verteidigungs-Dokument

Hauptamt Verwaltungr und Wirtschaft

SS-Wirtschafts-Verwaltungshauptamt 
Dienstgrade der SS-Führer

Ustf. Untersturmführer (entspricht dem Leutnant bei der Wehrmacht)

Ostf. Obersturmführer (Oberleutnant)

Hstf. Hauptsturmführer (Hauptmann)

Stbf. Sturmbannführer (Major)

Ostbf. Obersturmbannführer (Oberstleutnant)

Staf. Standartenführer (Oberst)

Of. Oberführer

Brif. Brigadeführer (Generalmajor)

Gruf. Gruppenführer (Generalleutnant)

Ogrf. Obergruppenführer (General) 
In der historischen Literatur fehlt noch immer eine umfassende Geschichte der SS, welche die Entwicklung dieser nationalsozialistischen Organisation, ihr Wesen und ihre Wirksamkeit in den verschiedensten Bereichen der Partei, des Staates und der Gesellschaft aufgrund der breiten heute vorliegenden Quellenbasis darstellt ${ }^{1}$. Der Gegenstand eines solchen Unternehmens ist so umfassend und vielseitig, die Probleme im einzelnen sind so kompliziert, daß diese Aufgabe wohl erst dann befriedigend gelöst werden kann, wenn einzelne Teilgebiete noch eingehender untersucht worden sind.

In der Aufhellung eines solchen, in der zeitgeschichtlichen Literatur bislang kaum behandelten Bereichs der SS-Aktivität liegt der Sinn der vorliegenden Arbeit, die sich mit den wirtschaftlichen Unternehmungen der SS befaßt. Die Gründung, der Erwerb und die Ausweitung von Handels- und Produktionsbetrieben durch die SS lassen ihre Kompetenz- und Machtexpansion im Gesamtgefüge des Dritten Reiches, unter der man gewöhnlich (nur) die Akkumulation administrativer, exekutiver und politischer Vollmachten versteht, unter einem neuen Aspekt erscheinen. Die Arbeit führt damit zugleich hinein in den historisch noch wenig erforschten Bereich des Wirtschaftslebens im Dritten Reich. Sie wird zu zeigen haben, wie die „bürgerliche“ Sphäre der Privatwirtschaft zwar formell respektiert wurde, tatsächlich aber unterwandert zu werden begann, indem eine totalitäre Herrschaftsorganisation die Normen und Möglichkeiten des Handelsrechts adaptierte.

Unter der ehrgeizigen Führung des „Reichsführers-SS“ Heinrich Himmler hatte sich die aus den "Schutzstaffeln " der Kampfzeit hervorgegangene SS, die ihrer rechtlichen Stellung nach immer noch eine Gliederung der NSDAP war, praktisch zu einer autonomen Macht neben Partei und Staat entwickelt, die gegen Ende des Krieges die entscheidenden Schlüsselpositionen in der Hand hatte und im Begriffe war, „Staat im Staate" zu werden. Sie beherrschte die gesamte Polizei (insbesondere die Geheime

1 Die in der „Schriftenreihe des Institutes für wissenschaftliche Politik in Marburg/Lahn" (herausgegeben von Prof. W. Abendroth) 1956 erschienene Dissertation von Ermenhild Neusüß-Hunkel: „Die SS“ hat lediglich gedruckte Quellen ausgewertet und das reichhaltige Dokumentenmaterial der zwölf Nürnberger Nachfolgeprozesse unberücksichtigt gelassen. Vom Standpunkt des Historikers aus erscheinen die in dieser „politisch-soziologischen Analyse" von außen herangetragenen Begriffe und Kategorien oft problematisch und manchmal abwegig. Noch unbefriedigender ist das Buch von Gerald Reitlinger: „Die SS, Tragödie einer deutschen Epoche", 1957 (Titel der englischen Originalausgabe : "The SS, Alibi of a Nation"), das mit seinen vorschnellen Verallgemeinerungen und zahlreichen ungenauen und falschen Angaben wissenschaftlichen Ansprüchen kaum genügen kann. 
Staatspolizei), mit der sie durch Personal- und Realunion eng verbunden war; sie verfügte mit dem SD ("Sicherheitsdienst des Reichsführers-SS“) über einen umfassenden politischen Nachrichten- und Abwehrdienst; ihr unterstand die Verwaltung und Bewachung der Konzentrationslager; sie hatte maßgeblichen Einfluß auf die Volkstums- und Siedlungspolitik in den eingegliederten und besetzten Ostgebieten; sie besaß in der Waffen-SS eine eigene Kampftruppe, die gegen Ende des Krieges etwa 600000 Mann zählte; sie hatte eine eigene Gerichtsbarkeit, eine eigene Verwaltungsbürokratie, eine eigene Ärzteschaft, eigene Forschungsinstitute, eigene Schulen, eigene Wohnsiedlungen, ein eigenes Bauwesen u.a.m. Mit der äußeren Machtstellung verband sich ideologisch der Anspruch, eine nationalsozialistische rassische Elite, ein „Orden guten Blutes“, eine zur Herrschaft berufene Führungsschicht zu sein.

Innerhalb des Gesamtumkreises der SS-Tätigkeit sind die wirtschaftlichen Unternehmungen wohl am wenigsten bekannt. Die Vorgänge auf diesem Gebiet spielten sich - da sie zum großen Teil mit dem Konzentrationslagerwesen zusammenhingen weitgehend $\mathrm{im}$ verborgenen $\mathrm{ab}$, und die SS tat alles, um sie nicht mehr als nötig publik zu machen. So erklärt es sich, daß nicht nur die breite Öffentlichkeit, sondern selbst ein großer Teil der SS-Angehörigen keine Kenntnis von der Existenz SS-eigener Wirtschaftsunternehmungen hatte. Über das Verhältnis der "Gesamt-SS“ zu ihren Einzelfunktionen, auch zu den Wirtschaftsunternehmen, äußerte sich Himmler in einer Ansprache an das Offizierkorps der Leibstandarte-SS „Adolf Hitler “ am 7. September 1940:

„Denn leben wird diese Waffen-SS nur dann, wenn die Gesamt-SS lebt. Wenn das gesamte Corps wirklich ein Orden ist, der . . . sich darüber klar ist, daß ein Teil ohne den anderen nicht denkbar ist. Ihr seid nicht denkbar ohne die Allgemeine SS, diese ist nicht denkbar ohne Euch. Die Polizei ist nicht denkbar ohne die SS, wir sind aber auch nicht denkbar ohne diese Exekutive des Staates, die in unser[er] Hand ist . . . wir sind nicht denkbar ohne die wirtschaftlichen Unternehmungen. Ein Teil, der Ihnen, den meisten, unbekannt sein muß, weil noch nie darüber gesprochen wurde . . ."

Aus der NS-Zeit gibt es keine Veröffentlichungen über die SS-Wirtschaftsbetriebe, und auch in der nach dem Kriege erschienenen einschlägigen Literatur werden sie - wenn überhaupt - nur am Rande erwähnt.

Umfang und Vielfalt dieser wirtschaftlichen Unternehmungen waren erstaunlich groß. Gegen Ende des Krieges besaß die SS mehr als 40 verschiedene Unternehmungen mit insgesamt etwa 150 Werken und Betrieben. Sie betätigten sich in den verschiedensten Geschäftszweigen (Steine und Erden, Lebensmittel und Getränke, Holz- und Eisenbearbeitung, Land- und Forstwirtschaft, Textil- und Lederverarbeitung, Buch- und Bildverlag u. a.m.). Diese Unternehmen standen alle unter der Dienstaufsicht und Verwaltung des von SS-Obergruppenführer Pohl geleiteten SSWirtschafts-Verwaltungshauptamtes (WVHA) und waren diesem auch organisatorisch und personell eingegliedert. Sie hatten zumeist die rechtliche Form von Han-

2 Dokument PS-1918 (IMT, XXIX, S. 98 ff.). 
delsgesellschaften ( $\mathrm{GmbH}$ oder $\mathrm{AG}$ ) und wurden $1940 \mathrm{zu}$ einem großen Konzern (Deutsche Wirtschaftsbetriebe $\mathrm{GmbH}$ ) zusammengeschlossen.

Ein großer Teil der SS-Wirtschaftsbetriebe stand von Anfang an in enger Verbindung mit den Konzentrationslagern. Die Verwendung von Häftlingen als Arbeitskräfte trifft zwar nicht für alle Unternehmen der SS zu, sie gibt aber gerade den größten und wichtigsten Firmen ihr charakteristisches Gepräge. Die Zwangsarbeit der KZ-Häftlinge, die während des Krieges ins Riesenhafte anschwoll, reichte jedoch andererseits über den engeren Bezirk der SS-Wirtschaftsbetriebe weit hinaus. Sie wird in unserer Darstellung nur im Zusammenhang der Wirtschaftsaktivität der SS, im Hinblick auf die spezifisch wirtschaftlichen Aspekte des Häftlingseinsatzes in ihren Betrieben (und die daraus sich ergebende Akzentverlagerung des gesamten Konzentrationslagerwesens, von einem primär politisch-polizeilichen zu einem Zwangsarbeits-Instrument) behandelt werden können. Dagegen müssen die vielfältigen sozialen und menschlichen Probleme der Häftlingszwangsarbeit besonderer Untersuchung überlassen bleiben.

Wegen ihrer Verbindung mit den Konzentrationslagern waren die SS-Wirtschaftsunternehmen Gegenstand einer langen Auseinandersetzung in dem von März bis November 1947 durchgeführten Prozeß gegen Pohl und seine Mitarbeiter vom WVHA, einem der zwölf Nürnberger Prozesse vor amerikanischen Militärgerichtshöfen. Unter den Angeklagten dieses Prozesses (Fall IV) befanden sich auch die leitenden Männer der SS-Wirtschaft: außer Pohl die früheren SS-Führer G. Lörner, H. Baier, Dr. Volk, K. Mummenthey, Dr. Bobermin, H. Klein und der Wirtschaftsprüfer Dr. Hohberg. In dem umfangreichen Dokumentenmaterial, das irn Zusammenhang mit diesem und den anderen Nürnberger Prozessen aus den alliierten Beutearchiven nach Deutschland gelangte, findet sich gerade zum Thema „SS-Wirtschaftsbetriebe" eine überraschend große Fülle von Material. Die hier vorgelegte Arbeit stützt sich im wesentlichen auf diese (vor allem in den Dokumentenserien NO und NI befindlichen) Nürnberger Dokumente und darüber hinaus auf die Aussagen und eidesstattlichen Erklärungen (Affidavits) der Angeklagten und Zeugen des Pohl-Prozesses. Es handelt sich, von wenigen Ausnahmen abgesehen, durchweg um ungedrucktes Quellenmaterial, das dem Verfasser in der zeitgeschichtlichen Sammlung des Instituts für Völkerrecht der Universität Göttingen (die seit November 1958 dem Staatlichen Archivlager Göttingen angegliedert ist) zugänglich war.

Die vorliegende Darstellung ist aus einer Dissertation hervorgegangen, die 1959 bei der Philosophischen Fakultät Göttingen eingereicht wurde. Mein besonderer Dank gilt an dieser Stelle meinem Göttinger Lehrer, Herrn Professor Dr. Percy Ernst Schramm, Herrn Dr. Hans-Günther Seraphim vom Staatlichen Archivlager Göttingen, der diese Untersuchung anregte, und Herrn cand. phil. Werner Präg, der das Register besorgte. 
Enno Georg, Schüler des Göttinger Historikers Percy Ernst Schramm, gibt umfassenden Einblick in einen weithin unbekannten Aktionsbereich der SS im Staate Hitlers. SS-eigene Wirtschaftsunternehmen entstanden, vor allem auf dem Sektor des Baugewerbes, schon vor 1939 vor allem in Gestalt von Häftlingsbetrieben, d. h. als Nebenwirkung des Konzentrationslager-Systems der SS. Frühzeitig fand Himmlers Bestreben nach Ausweitung der SS-Kompetenzen hier ein neues Betätigungsfeld. Zugleich bot sich dem Reichsführer SS dabei die Möglichkeit, weltanschauliche Lieblingsvorstellungen sowie bestimmte technische Erfindungen und Experimente in eigener Regie zu verwirklichen. Der dann im Krieg anschwellende Konzern der SS-Betriebe, Deutsche AusrüstungsWerke (DAW), Deutsche Erd- und Steinwerke (DEST) u. a. m., die in die Form privatkapitalistischer $\mathrm{GmbHs}$ gekleidet waren, machte das Wirtschaftsverwaltungshauptamt der SS schließlich zu einem bedeutenden Faktor der Rüstungswirtschaft. Es zeichnete sich hierbei auch die Unterwanderung der privaten Konkurrenz. wirtschaft durch eine letztlich nicht nach Rentabilitätsgesichtspunkten arbeitende, aufgrund der Staatssklaverei in den Konzentrationslagern von Lohnverpflichtungen unabhängige SS-Monopolwirtschaft ab. Der Verfasser zeigt auch, welche Zukunftsvorstellungen Himmler mit diesem Instrument SSeigener Wirtschaft verband. 
Schriftenreihe der Vierteljahrshefte für Zeitgeschichte

IN VORBEREITUNG SIND:

LADISLAUS HORY MARTIN BROSZAT

Der Kroatische Ustascha-Staat 1941-1945

Ein dokumentarischer Bericht aus Erinnerungen und deutschen Akten

ROLF GEISSLER

\section{Dekadenz und Heroismus}

Zeitroman und völkischnationalistische Literaturkritik

(1919-1936)

Die Ideologie der spanischen Falange

Dargestellt an ausgewählten Texten José Antonio Primo de Riveras

\section{Die Gleichschaltung der Preufischen Akademie der Schönen Künste 1933}

Dokumente und Zeugnisse Herausgegeben von Hildegard Brenner

Jährlich erscheinen zwei

Veröffentlichungen 